http://jmscr.igmpublication.org/home/ ISSN (e)-2347-176x ISSN (p) 2455-0450

crossref DOI: https://dx.doi.org/10.18535/jmscr/v7i10.54

Journal Of Medical Science And Clinical Research

IGM Publication

An Official Publication of IGM Publication

\title{
Comparison of Antibiotic Profiles of Methicillin Sensitive and Methicillin Resistant Staphylococcus aureus Isolated from Different Specimens of Hospitalized Patients in Dhaka City
}

\author{
Authors \\ Dr Hafiza Sultana ${ }^{*}$, Dr Humayun Sattar ${ }^{2}$, Dr Shirin Tarafder ${ }^{3}$, \\ Dr Jogendra Nath Sarker ${ }^{4}$, Dr Md. Tofael Hossain Bhuiyan ${ }^{5}$, \\ Dr Md. Mostaqimur Rahman ${ }^{1}$, Dr Md. Abdullah Yusuf ${ }^{6}$
}

${ }^{1}$ Assistant Professor, Department of Microbiology, Rangpur Medical College, Rangpur, Bangladesh

${ }^{2}$ Professor, Dept. of Microbiology \& Immunology, BSMMU, Dhaka, Bangladesh

${ }^{3}$ Associate Professor, Dept. of Microbiology \& Immunology, BSMMU, Dhaka, Bangladesh

${ }^{4}$ Assistant Professor, Department of Microbiology, Dinajpur Medical College, Dinajpur, Bangladesh

${ }^{5}$ Professor \& Head, Department of Neurosurgery, Rangpur Medical College Hospital, Rangpur, Bangladesh

${ }^{6}$ Assistant Professor, Department of Microbiology, National Institute of Neurosciences and Hospital, Dhaka,

Bangladesh

*Corresponding Author

Dr Hafiza Sultana

\begin{abstract}
Background: Staphylococcus aureus, resistant to methicillin, has appeared as a pathogen connected with diseases acquired in hospitals and in the community globally.

Objective: The objective of this study was to compare the methicillin-sensitive and methicillin-resistant Staphylococcus aureus isolated from the different samples of hospitalized patients.

Methodology: This cross-sectional study was carried out in the Department of Microbiology and Immunology at Bangabandhu Sheikh Mujib Medical University, Dhaka from January 2010 to December 2010 for a period of one (01) year. All patients admitted to hospital were chosen as study population with distinct kinds of diseases. These patients gathered different clinical samples depending on the locations of the diseases. Staphylococcus aureus (S. aureus) was isolated and identified through staining, cultivation and biochemical testing. To detect the methicillinresistant Staphylococcus aureus, an oxacillin disk diffusion test was performed. PCR wa used to detect the MRSA mecA gene.

Results: Over the period from January 2010 to December 2010, a total of 120 Staphylococcus aureus (S. aureus) isolates were gathered from 266 samples from three hospitals. Patients with age group 61 to 70 years had the largest proportion of incidence of MRSA. The patterns of antimicrobial susceptibility of 19 MRSA strains were reported on disk diffusion. Multi-resistant among them were 14 (73.7 percent) and non-multi-resistant strains were 5 (26.3 percent). Other than the reserve resorts of drugs gentamycin 14(73.7\%) were most sensitive followed by cotrimoxazole 11(57.9\%), ciprofloxacin 6(31.6\%), erythromycin 6(31.6\%) and azithromycin 5(26.3\%). The site of infection of MRSA was categorized in seven categories depending on the type of specimens. Here skin and soft tissue infection by MRSA strains were predominant and it was 10(14.3\%). The incidence of MRSA in three different hospitals was recorded. In BSMMU the incidence was 11 (13.75\%), in DMCH 2 (20.0\%) and in Popular Diagnostic Center it was $6(20.0 \%)$.

Conclusion: In conclusion, majority MRSA are multiresistant but sensitive towards vancomycin and linezolid.

Keywords: Methicillin sensitive;methicillin resistant; Staphylococcus aureus; hospital-acquired.
\end{abstract}




\section{Introduction}

Staphylococcus aureus is a hospital as well as community-acquired bacteria which causes a broad spectrum of diseases and this is ranging from skin and soft tissue infections to endocarditis and fatal pneumonia ${ }^{1}$. The pathogenicity of these bacteria is associated with different enzymes and toxins which includes enterotoxins, exfoliative toxin, toxic shock syndrome toxin, and PantonValentine leucocidin ${ }^{2}$. This bacterium possess the remarkable ability to adapt to different types of antibiotics and now with the emergence of multidrug resistant (MDR) bacteria, S. aureus is a warning for public health ${ }^{3}$. Methicillin-resistant $S$. aureus (MRSA) strains are able to grow in the presence of methicillin, oxacillin and nafcillin ${ }^{4}$.

Resistance to methicillin in S. Initially, aureus was identified in Europe in the 1960s, just one year after methicillin was introduced ${ }^{5}$. MRSA isolates are discovered today not only in most countries ' hospitals, but also in societies, and are often resistant to multiple antibiotics ${ }^{6}$. Clinical infections are most common in patients in hospital intensive care units, nursing homes, and other chronic care facilities; however, MRSAs are emerging as an important community acquired pathogen as well. Although there are some reports on the prevalence of vancomycin resistant $S$. aureus (VRSA) and vancomycin intermediate $S$. aureus (VISA), most MRSA isolates are susceptible to vancomycin and teicoplanin; therefore, resistance increase to these antibiotics results in the limitation of treatment options and also the requirement of a new class of antibiotics ${ }^{7}$. The current research was conducted in this context to compare antibiotic profiles of methicillinsensitive and methicillin-resistant Staphylococcus aureus isolated from separate samples of hospitalized patients.

\section{Methodology}

This cross-sectional study was conducted in the Department of Microbiology and Immunology at Bangabandhu Sheikh Mujib Medical University, Dhaka from January 2010 to December 2010 for a period of twelve (12) months. Different clinical samples were gathered from patients of both sexes at any era, including wound swab, pus, blood, urine, tracheal aspiration, throat swab, sputum, nasal swab, elevated vaginal swab, burn swab, pleural effusion drain fluid and fluid.All samples were aseptically gathered from three hospitals, namely Bangabandhu Sheik Mujib Medical University (BSMMU), Dhaka Medical College Hospital (DMCH), Dhaka and Dhaka Private Diagnostic Center. The samples were inoculated into appropriate media and were incubated aerobically at $37^{0} \mathrm{C}$ for 24 hours. Then colonies were identified for Staphylococcus species and were confirmed by Gram staining, colony morphology, haemolytic status, pigment production, mannitol fermentation test, motility test (MIU) and other relevant biochemical tests like catalase test, coagulase test both slide and tube test as per standard procedure ${ }^{6}$. In this study, screening for MRSA was done by oxacillin and oxacillin screening agar. In case of oxacillin the diameter of zone of inhibition $\leq 10 \mathrm{~mm}$ was taken as resistant ${ }^{7}$. Conventional PCR was performed to detect $m e c A$ gene of 22 suspected $S$. aureus strains resistant to oxacillin by disc diffusion method at the Molecular Laboratory in the Department of Microbiology and National Forensic DNA Profiling Laboratory of Dhaka Medical College, Dhaka. Methicillin-resistant S. aureus (MRSA) strain [ATCC 43300] were used as positive control. PCR for mecA gene detection were performed by formation of bacterial pellet, DNA extraction, preparation of reaction mixture $(25 \mu \mathrm{l})$ and running in thermo cycler. Primers used for detection of the mecA gene producing a 309-bp amplicon were as follows:

mecA1-F- 5' TGGCTATCGTGTCACAATCG 3' (positions 885 to 905 ) and

mecA2-R- 5' CTGGAACTTGTTGAGCAGAG 3' (positions 1174 to 1194 )

PCR reactions were performed in a thermo cycler under the following conditions: initial denaturation for 10 minutes at $94^{\circ} \mathrm{C}$ followed by 30 cycles at $94^{\circ} \mathrm{C}$ for 1 minute, at $54^{\circ} \mathrm{C}$ for 1 
minute, then at $72^{\circ} \mathrm{C}$ for 1 minute. Final extension was for 7 minutes at $72^{\circ}$ C.Mixed the amplicon and ladder with dye (4-5:1 ratio). Then pipetting and dispensing were done onto the wells on gel made by comb. Start the gel electrophoresis at 100 volt for 60 minutes until the end of the reaction indicated by orange color advancement was over. Ethidium bromide $(7.5 \mu 1)$ mixed with distilled water $(100 \mathrm{ml})$. Gel was placed in this mixture for 30 minutes staining. Again destaining done in pure water for 20 minutes. The destained gel was placed on UV transilluminator and observed for the presence of DNA bands. Gels were visualized and photographed under ultraviolet illumination. Precautions were taken to prevent the samples from being contaminated by each other or by the skin of laboratory personnel.

\section{Results}

A total of 120 Staphylococcus aureus (S. aureus) isolates were collected from 266 specimens from three different hospitals. The highest percentage of MRSA occurrence was in patients having age group 61 to 70, next of which 51 to 60 years (Table 1).

Table 1: Age distribution of the patients having Staphylococcus aureus infection including MRSA in three different hospitals $(n=120)$

\begin{tabular}{|l|c|c|c|}
\hline Age Groups & MRSA & MSSA & Total \\
\hline 0 to 20 Years & $0(0.0 \%)$ & $28(100.0 \%)$ & $28(23.3 \%)$ \\
\hline 21 to 40 Years & $11(40.0 \%)$ & $44(80.0 \%)$ & $55(45.8 \%)$ \\
\hline 41 to 60 Years & $5(15.4 \%)$ & $21(84.6 \%)$ & $26(21.6 \%)$ \\
\hline 61 to 80 Years & $3(30.0 \%)$ & $8(70.0 \%)$ & $11(8.3 \%)$ \\
\hline Total & $\mathbf{1 9}(\mathbf{1 5 . 8 \%})$ & $\mathbf{1 0 1 ( 8 4 . 2 \% )}$ & $\mathbf{1 2 0}(\mathbf{1 0 0 . 0 \%})$ \\
\hline
\end{tabular}

The antimicrobial susceptibility patterns of 19 MRSA strains on disc diffusion were recorded.
Among them 14(73.7\%) were multi-resistant and $5(26.3 \%)$ were non-multi-resistant strains. Other than the reserve resorts of drugs gentamycin 14(73.7\%) were most sensitive followed by cotrimoxazole $11(57.9 \%)$, ciprofloxacin $6(31.6 \%)$, erythromycin $6(31.6 \%)$ and azithromycin $5(26.3 \%)$ (Table 2).

Table 2: Antimicrobial susceptibility pattern of MRSA strains $(n=19)$

\begin{tabular}{|c|c|c|}
\hline Antimicrobial agents & Sensitive & Resistant \\
\hline \multicolumn{3}{|l|}{ Penicillin } \\
\hline Penicillin & $0(0.0 \%)$ & $19(100.0 \%)$ \\
\hline Ampicillin & $0(0.0 \%)$ & $19(100.0 \%)$ \\
\hline Cloxacillin & $2(10.5 \%)$ & $17(89.5 \%)$ \\
\hline Oxacillin & $3(15.8 \%)$ & $16(84.2 \%)$ \\
\hline \multicolumn{3}{|l|}{ Cephalosporin } \\
\hline Cefoxitin & $0(0.0 \%)$ & $19(100.0 \%)$ \\
\hline Cephradin & $2(10.5 \%)$ & $17(89.5 \%)$ \\
\hline \multicolumn{3}{|l|}{ Aminoglycoside } \\
\hline Gentamycin & $14(73.7 \%)$ & $5(26.3 \%)$ \\
\hline \multicolumn{3}{|l|}{ Quinolone } \\
\hline Ciprofloxacin & $6(31.6 \%)$ & $13(68.4 \%)$ \\
\hline \multicolumn{3}{|l|}{ Carbapenem } \\
\hline Imipenem & $19(100.0 \%)$ & $0(0.0 \%)$ \\
\hline \multicolumn{3}{|l|}{ Macrolid } \\
\hline Erythromycin & $6(31.6 \%)$ & $13(68.4 \%)$ \\
\hline Azithromycin & $5(26.3 \%)$ & $14(73.7 \%)$ \\
\hline \multicolumn{3}{|l|}{ Lymphosamide } \\
\hline Clindamycin & $17(89.4 \%)$ & $2(10.6 \%)$ \\
\hline \multicolumn{3}{|c|}{ Sulphonamide+Trimeth. } \\
\hline Cotrimoxazole & $11(57.9 \%)$ & $8(42.1 \%)$ \\
\hline \multicolumn{3}{|l|}{ Glycopeptide } \\
\hline Vancomycin & $19(100.0 \%)$ & $0(0.0 \%)$ \\
\hline \multicolumn{3}{|l|}{ Oxazolidinon } \\
\hline Linezolid & $19(100.0 \%)$ & $0(0.0 \%)$ \\
\hline
\end{tabular}

The site of infection of MRSA was categorized in seven categories depending on the type of specimens. Here skin and soft tissue infection by MRSA strains were predominant and it was 10(14.3\%) (Table 3).

Table 3: Distribution of MRSA isolates according to site of infection $(n=120)$

\begin{tabular}{|l|c|c|c|c|}
\hline Sites of infection & MRSA & MSSA & Total & \multirow{2}{*}{ P Value } \\
\hline Blood stream infection & $3(50.0 \%)$ & $3(50.0 \%)$ & $6(100.0 \%)$ & \\
\hline Respiratory tract infection & $1(100.0 \%)$ & $0(0.0 \%)$ & $1(100.0 \%)$ & \\
\hline Urinary tract infection & $3(10.0 \%)$ & $27(90.0 \%)$ & $30(100.0 \%)$ & \\
\cline { 1 - 4 } Skin and soft tissue infection & $10(14.3 \%)$ & $60(85.7 \%)$ & $70(100.0 \%)$ & \multirow{2}{*}{0.001} \\
\cline { 1 - 4 } Genital tract infection & $1(50.0 \%)$ & $1(50.0 \%)$ & $2(100.0 \%)$ & \\
\cline { 1 - 2 } ENT infection & $1(11.1 \%)$ & $8(88.9 \%)$ & $9(100.0 \%)$ & \\
\cline { 1 - 3 } Other body fluid infection & $0(0.0 \%)$ & $2(100.0 \%)$ & $2(100.0 \%)$ & \\
\hline Total & $19(15.8 \%)$ & $101(84.2 \%)$ & $120(100.0 \%)$ & \\
\hline
\end{tabular}


The incidence of MRSA in three different hospitals was recorded. In BSMMU the incidence was $11(13.75 \%)$, in $\mathrm{DMCH} 2(20.0 \%)$ and in
Popular Diagnostic Center it was 6 (20.0\%) (Table 4).

Table 4: Incidence of MRSA and MSSA isolates in three different hospitals $(n=120)$

\begin{tabular}{|l|c|c|c|c|}
\hline Name of Hospitals & MRSA & MSSA & Total & P value \\
\hline BSMMU & $11(13.75)$ & $69(86.25)$ & $80(100.0 \%)$ & \\
\cline { 1 - 4 } DMCH & $2(20.0)$ & $8(80.0)$ & $10(100.0 \%)$ & \multirow{2}{*}{0.0024} \\
\hline Private Diagnostic Centre & $6(20.0)$ & $24(80.0)$ & $30(100.0 \%)$ & \\
\cline { 1 - 4 } Total & $\mathbf{1 9}(\mathbf{1 5 . 8})$ & $\mathbf{1 0 1}(\mathbf{8 4 . 2})$ & $\mathbf{1 2 0}(100.0 \%)$ & \\
\hline
\end{tabular}

\section{Discussion}

S. Aureus is the leading cause of infection in hospitalized patients and the second leading cause of outpatient infection ${ }^{8}$. Today, $S$. aureus is the leading cause of nosocomial pneumonia and the second leading cause of bloodstream infections in the world ${ }^{9}$. MRSA is also dominant in intensive care unit (ICU) of hospitals in most parts of the world $^{10}$.

Around 20.0 percent of healthy individuals are persistent S carriers. Aureus and 60.0 percent are carriers that are intermittent. In patients with haemodialysis, illicit injection of drugs, surgical patients and patients with insulin reliance or poorly monitored diabetes, colonization rates are improved $^{11}$. Healthcare-related infection is the major cause of morbidity and mortality 12 and the appearance and fast spread of antimicrobial resistance among the floating organisms in the hospital has critically influenced the management of all these conditions. The significance of MRSA identification, therefore, is particularly important for therapeutic and epidemiological purposes. Hence, the methods used to detect MRSA in clinical samples should have high sensitivity and specificity and most importantly the result should be available within a short time ${ }^{13}$.

A total of 120 Staphylococcus aureus were isolated from 266 clinical specimens from three hospitals of which $80(66.7 \%)$ S. aureus were collected after prior confirmation from the department of Microbiology \& Immunology laboratory of Bangabandhu Sheikh Mujib Medical University (BSMMU), 10 (8.3\%) from Dhaka Medical College Hospital (DMCH) and rest
$30(25.0 \%)$ were collected from Private Diagnostic Center, Dhakato get a more representative picture of the situation. These isolates were subjected to antimicrobial susceptibility testing by oxacillin and cefoxitin and PCR for detection of the mecA gene. The rate of MRSA infection in different age group in comparison to MSSA infection found that, MRSA infection rate increased gradually with age. It was highest in age group 61-70 years (30\%) and next of which 51-60 years $(23.1 \%)$ where as in the age group 0-10 years and 11-20 years the MRSA rate was $0 \%$, This result is in conformity with the reports of Khurram et $\mathrm{al}^{14}$ from Pakistan, Lepelletier et $\mathrm{al}^{15}$ (2004) from France and Sadoyama et $\mathrm{al}^{16}$ from Brazil. They revealed that MRSA infection was considerably greater in elderly patients, and this may be due to the reality that elderly individuals are more exposed to antimicrobial agents, which lead to selective antibiotic stress and growth of resistant strain infection and, in relation to decreasing immunity in ancient age, increases the danger of MRSA strain infection, which could be the reason for elevated strain infection.

Therefore, number of methicillin and cefoxitin resistant and sensitive strains isolated by this technique were $17(14.2 \%)$ and 103(85.8\%), $19(15.8 \%)$ and $101(84.2 \%)$ respectively. There is a significant difference in resistance pattern of oxacillin and cefoxitin by disc diffusion method, while vancomycin $117(97.5 \%)$ and linezolid showed $120(100 \%)$ sensitivity.Bukhari ${ }^{17}$ reported $41.9 \%$ resistance against oxacillin from 1102 isolates in compare to our $15.8 \%$ from 120 isolated S. aureus. Other antibiotics like penicillin 
$1056(95.8 \%)$ and cotrimoxazole 699(63.6\%) resistance is higher than the present study. Study by Subedi and Brahmadathan ${ }^{18}$ reported 18 $(15.4 \%)$ methicillin-resistance which is in agreement with that of the present study.

The prevalence of MRSA in hospitals varies considerably from one region to another and among hospitals in the same area ${ }^{17}$. This variation ranges from 2-61\% as stated by Hafiz et $\mathrm{al}^{9}$ Prevalence of MRSA as reported by Safdar et $\mathrm{al}^{10}$ indicates $<10 \%$ to $65 \%$. In India it ranges from 30 to $70 \%{ }^{11}$. According to Sturenburgh et al ${ }^{19}$ MRSA accounts for at least 15.0 to $20.0 \%$ of clinical isolates of $S$. aureus. Subedi and Brahmadathan $^{18}$ published their MRSA percentage $15.4 \%$ in their research note. From their experience it can be assume that such variation may also exists between our hospitals and health care facilities. MRSA isolates among the three hospitals is recorded but show little variation regarding MRSA which were 11(13.8\%) in BSMMU, 2(20.0\%) in DMCH and 2(20.0\%) PDC. If larger number of samples could have been included in this study then the picture would have been different.

The prevalence rate of MRSA is $15.8 \%$ in the present study and is similar or within the range of almost all above studies. Low prevalence rate can be explained by the fact that they were conductedon isolates of $S$. aureus collected from relatively less ill persons while high prevalence rates were available on studies done on severely ill patients ${ }^{12}$. Infection prevention measures are enforced in BSMMU for prevention of nosocomial infection and in the private hospitals the patients themselves and the management of the hospital both are aware of factors that may be a source of infection to their patients, this may explain low prevalence of MRSA among our cases. Review of numerous other research in Bangladesh shows a growing trend of MRSA across the nation. A multi-center research with four divisional clinics at Ibrahim Medical College (Haq, 2009) revealed an MRSA rate variety of 3263 percent.
In the current study, among the 19 MRSA strains $14(73.7 \%)$ were multi-resistant strains and $5(26.3 \%)$ were non-multi-resistant.mMRSA are defined as MRSA resistant to two or more classes of antibiotics in addition to $\beta$ lactams;mMRSAaccounts for $75.0 \%$ of isolations in 2001.Literatures suggest HA-MRSA strains tend to be more multidrug resistant in contrast to CA-MRSA strain with hallmark resistance to fluoroquinolones. The distribution of MRSA by the site of infection was as follows; 3(50.0\%) blood stream, 10(14.3\%) skin and soft tissue, $1(50.0 \%)$ genital tract and $1(11.1 \%)$ ENT. Only $1(100.0 \%)$ strain of respiratory tract infection yielded MRSA which was collected from laboratory stock culture. No MRSA was detected from body fluids other than blood. Maximum number of MRSA was isolated from Skin soft tissue followed by blood stream and urinary tract infections. From 57 ICU specimens 7(12.3\%) were S.aureus isolates of which $4(57.1 \%)$ came out to be MRSA. If larger number of ICU sample could have been collected the real situation of MRSA related infection could have been possible.MRSA accounts for $>50 \%$ of S. aureus isolates from patients in ICUs in the NNIS system; in 2003, 59.5\% of S.aureus isolates in NNIS ICUs were MRSA (NNIS, 2003). By 2004, this figure increased to $63 \%$ (CDC, 2006).

MSSA and MRSA isolates' pattern of antibiotic susceptibility varied considerably. MSSA isolates were prone to most of the medicines tested, although some resistance was noted to penicillin, tetracycline, tobramycin and, to some extent, clindamycin, amikacin and kanamycin. Multiple drug resistance was prevalent in the event of MRSA, however, and only a few antibiotics were effective against these isolates. MRSA strains have been discovered to be more resistant than MSSA strains to other antibiotics. In the case of erythromycin, ciprofloxacin, tobramycin, tetracycline, gentamicin, and amikacin, significant difference $(\mathrm{p}<0.05)$ was noted. There was a important distinction between MRSA sensitivity patterns and MSSA isolates patterns. 


\section{Conclusion}

According to antibiotic profiles, the bulk of MRSA are multi-resistant. All strains, however, are susceptible to vancomycin and linezolid. Skin and soft tissue infection is the most prevalent location of MRSA infection. A large-scale multicenter survey should be conducted to assess Bangladesh's actual condition.

\section{References}

1. Akpaka PE, Monecke S, Swanston WH, Rao AC, Schulz R, Levett PN. Methicillin sensitive Staphylococcus aureus producing Panton-Valentine leukocidin toxin in Trinidad \& Tobago: a case report. J Med Case Rep 2011;5:157

2. Gordon RJ, Lowy FD. Pathogenesis of methicillin-resistant Staphylococcus aureus infection. Clin Infect Dis 2008;46 Supp15:S350-9

3. Diep BA, Chambers HF, Graber CJ, Szumowski JD, Miller LG, Han LL, et al. Emergence of multidrug-resistant, community-associated, methicillinresistant Staphylococcus aureus clone USA300 in men who have sex with men. Ann Intern Med 2008;148(4):249-57

4. Rahimi F, Bouzari M, Katouli M, Pourshafie MR. Antibiotic resistance pattern of methicillin resistant and methicillin sensitive Staphylococcus aureus isolates in Tehran, Iran. Jundishapur Journal of Microbiology. 2013;6(2):144-9.

5. Zetola N, Francis JS, Nuermberger EL, Bishai WR. Community-acquired meticillin-resistant Staphylococcus aureus: an emerging threat. Lancet Infect Dis 2005;5(5):275-86

6. Turlej A, Hryniewicz W, Empel J. Staphylococcal cassette chromosome mec (Sccmec) classification and typing methods: an overview. Pol J Microbiol. 2011;60(2):95-103
7. Howden BP, Davies JK, Johnson PD, Stinear TP, Grayson ML. Reduced vancomycin susceptibility in Staphylococcus aureus, including vancomycin-intermediate and heterogeneous vancomycin-intermediate strains: resistance mechanisms, laboratory detection, and clinical implications. Clin Microbiol Rev 2010;23(1):99-139

8. Styers D, Sheehan DJ, Hogan P, Sahm DF. Laboratory-based surveillance of current antimicrobial resistance patterns and trends among Staphylococcus aureus: 2005 status in the United States. Ann Clin Microbiol Antimicrob2006;5:2

9. Hafiz S, Hafiz AN, Ali L, Chughtai AS, Memon B, Ahmed A et al.Methicillin resistant Staphylococcus aureus: a multicenter study. J Pak Med Assoc 2002; 52: 312-315

10. Safder N, Narans L, Gordon B, Maki DG. Comperison of culture screening methods for for detection of nasal carriage of methicillin-resistant Staphylococcus aureus: a prospective study comparing 32 methods. J Clin Microbiol2003;41:316366

11. Anwar MS, Jaffery G, Bhatti KU, Tayyab M,Bokhari SR. Staphylococcus aureus and nasal carriage in general population. J Coll Physic Sur Pak2004; 4:661-664.

12. Shahidullah MS, Yusuf MA, Khatun Z, Ara UK, Mitul MT. Antibiotic Sensitivity Pattern of Bacterial Isolates from Different Clinical Specimens: Experience at NICVD, Dhaka. Cardiovascular J 2012;5(1):67-72

13. Yusuf MA, Sattar AA, Habib ZH, Roy S. Clinical and Diagnostic Significance of Methicillin-resistant Staphylococcus aureus Infection in Bangladesh: A Systematic Review. British Microbiol Res J 2014;4(7):785.

14. Khurram IM, Khan SA, Khawja AA, Khan R, Khokher SA,Khawar $S$ et al. Risk 
factors for clinical infection in patients colonized with methicillin-resistant Staphylococcus aureus (MRSA). J Pak Med Assoc 2004;54 (8): 408-412

15. Lepelletier D, Ferreol S, Villers D, Richet H. Methicillin-resistant Staphylococcus aureus nosocomial infection in ICU: risk factors, morbidity and cost. Pathol Biol 2004;52(8): 474-479

16. Sadoyama G and Filho PP. 2000. Risk factors for Methicillin-resistant and sensitive Syaphylococcus aureus infection in Brazilian University Hospital. Braz J Infect Dis 2000;4(3):135-143

17. Bukhari SZH. Trends in antimicrobial resistance and occurrence of methicillin resistant Staphylococcus aureus (MRSA): molecular basis and clinical implications. [PhD thesis]. Department of Microbiology, Quaid-i-Azam University, Islamabad, Pakistan, 2006

18. Subedi S, Brahmadathan KN. Antimicrobial susceptibility patterns of clinical isolates of Staphylococcus aureus in Nepal. Clin Microbiol Infect 2005; 11:235-237

19. Sturenburg E. Rapid detection of methicillin-resistant Staphylococcus aureus directly from clinical samples: methods, effectiveness and cost considerations. German Med Sci 2009;7:1612-616. 